\title{
Some quantitative evaluations on finite difference local and global results
}

\author{
Eugenio Costamagna \\ University of Pavia \\ via Sabotino 11/23, 16156 Genova, Italy \\ e-mail:eugenio.costamagna@unipv.it
}

(Received: 10.06.2015, revised: 26.11.2015)

\begin{abstract}
Refined Schwarz-Christoffel (SC) conformal transformations allow us to perform reliable quantitative evaluation of the accuracy of local computation of electric and magnetic fields with limited effort, which can be useful to complement well known comparisons of global results. In this paper some examples are presented for mesh point potentials obtained by means of finite difference (FD) methods, but it is possible that similar considerations will be useful in the case of finite element methods (FEM) or meshless computations too.
\end{abstract}

Key words: finite difference, Schwarz-Christoffel, static fields

\section{Introduction}

The availability of powerful methods for numerical computation and inversion of the Schwarz-Christoffel (SC) formula allows us to compare with limited computational effort not only global quantities, as capacitances in the following FD examples, but local results too, as mesh point potentials [1-4]. The main usefulness is to cope with the field singularities caused by sharp corners in polygonal boundaries, both when dealing with plane or rotational symmetry geometries, and with internal or external domains: recent examples of results are reported in [5-7]. In fact, the following calculations concern well known case studies, but the author is not aware of similar studies related to the accuracies of single point potentials or to the improvement of capacitance evaluations in general conditions.

\section{An L-shaped domain}

Considering two equal side dihedral electrodes and side magnetic walls as in Fig. 1, a very good reference value for the capacitance is easily obtained by means of numerical inversion (SCNI) of the Schwarz-Christoffel conformal transformation [1,2]. The geometry was already used as a test problem when introducing a novel approach to the SC formula calculations [3]. 
The geometry with short sides in Fig. 1 was selected to enhance the effects of the electrode shape on the field behaviour, avoiding parallel plate effects. The domain was mapped into a rectangle via SCNI, and a capacitance in air of about $C_{\mathrm{SC}}=2.265354 \cdot 10^{-11} \mathrm{~F}$ was found. A vertical line is traced in the figure, and both the rectangle and the transformed map of the vertical line are shown in Fig. 2. Solid lines indicate conductors, dashed lines Neumann walls.

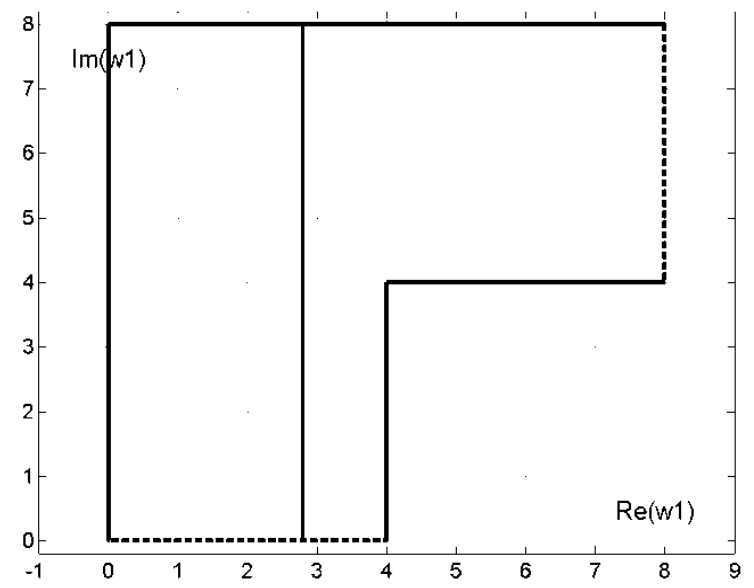

Fig. 1. An example of L-shaped domain, with a vertical straight line. Coordinates in arbitrary units

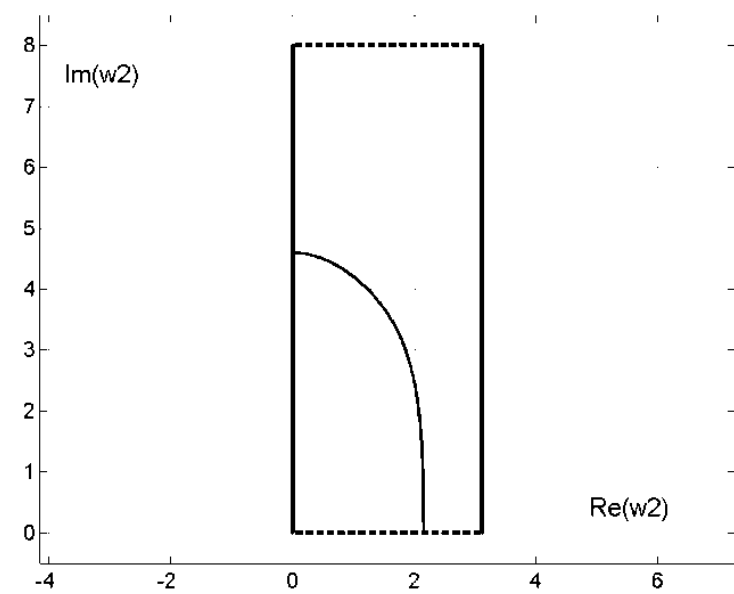

Fig. 2. The SC map of the domain in Fig. 1 into a rectangle, with the map of the vertical straight line. Coordinates in arbitrary units

For the same geometry, capacitances and mesh potentials have been obtained from a simple FD code performing a successive over-relaxation (SOR) process for various numbers of cells. With numbers of cells on each side on the large ("exterior") L ranging from 8 to 256 (and from 4 to 128 cells on each "interior" conductor side), the capacitance values obtained for the exterior conductor have been found to exceed $C_{\mathrm{SC}}$ by about $2.3 \%$ to some $0.02 \%$. 
This was expected, as no field singularities are found along this conductor. Instead, the absolute values of the errors on capacitances computed on the interior conductor ranged from more than $12 \%$ to about $1.4 \%$. In fact, the ratios between the external capacitance, $C_{\text {ext }}$, and the internal one, $C_{\mathrm{int}}$, range from more than $13 \%$ to about $1.5 \%$, and they are of about $5.5 \%$ with 32 cells on the long sides.

Now, if the vertical line of Fig. 1 coincides with a mesh vertical line, the true (i.e., the SC) potentials of the mesh points lying on it can be derived from the abscissas of the corresponding points on the map of Fig. 2 (to make easier any comparison, potentials will be always computed on points lying on the grid defined by the most dense mesh).

A comparison of the potentials obtained from FDs with those provided by SC for grid points adjacent to the interior conductor (used, of course, to evaluate dielectric fluxes) shows maximum potential ratios ranging from about $1.8 \%$ with 32 intervals to about $0.5 \%$ with 256 intervals (absolute values; the numbers of cells or intervals are always referred to the exterior conductor), which are by far the largest on the mesh, rapidly decaying going away from the vertex.

Observing the behaviours of the potentials involved in FD dielectric flux computations, it is evident that elemental integration rules are perfectly adequate: as the singularities of the field are not properly taken into account when computing the FD mesh potentials, no further problems are to be expected from their smoothed behaviours. Of course, all that is widely known, but a deeper comparison of the charge densities along the conductors obtained by FDs and the exact ones provided by SC seems to be worth of attention, looking for any possibility of validating use of known charge density models.

\section{Modeling charge densities}

Reliable models for charge densities are of paramount importance to enlighten not only the behaviours of simple SOR procedures, but also the known capabilities of FEM codes in order of capture the effects of boundary field singularities, and this investigation originates from a short discussion on the topic reported in [4]. Some aspects of the influence of the field singularities at the boundaries of capacitance geometries have been reported in [6] too.

Successful models have been proposed by Van Bladel in [8] and [9], both for plane and rotational symmetry geometry, and then used and compared in [10], were the field analysis was performed by means of SCNI of well matched geometries.

As already noted, the key problem is to limit the region neighboring a field singularity, in which to use the exponential Van Bladel's models, and to see if the influence of other singularities or other boundary segments is suggesting any adjustment of the model.

We start by observation of the FD and of the SC surface charge densities on the actual geometry of Fig. 1, which are shown in Fig. 3 and 4, but SCNI can provide similar data for geometries of virtually any shape and ratios among side lengths. Here, the SC value have been derived evenly scanning the original side in Fig. 1, taking into account the corresponding 
different lengths in Fig. 2 and assigning them to the corresponding grid intervals after suitable scaling.

The failure of the FD procedure in computing the charge densities is evident at the left hand of the figure, but it is equally evident the very good agreement with the SC results at the right, early starting from the left, at about $1 / 15$ of the side length.

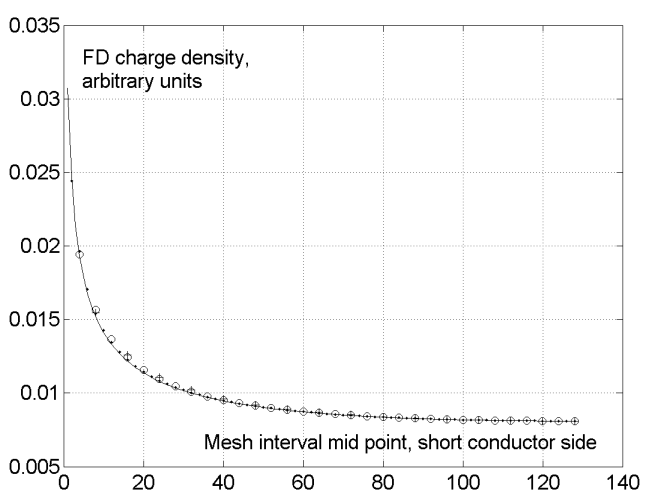

Fig. 3. Charge densities derived from the potentials on the FD mesh with various grid dimensions, from 32 to 256 meshes, showing in this case negligible differences in their behaviours

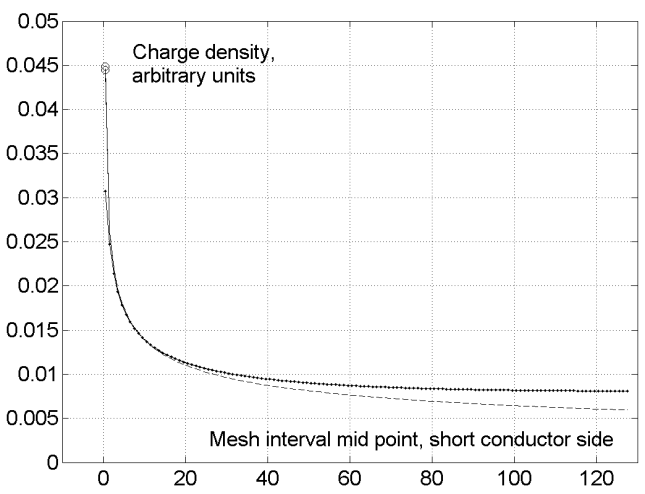

Fig. 4. FD charge densities (solid line and dots), SC charge densities (solid line and circle), and Van Bladel charge density model (dashed line and again a circle)

The Van Bladel model (with the singularity exponent $v$ of Fig. 4.4 in [8], page 120, equal to $2 / 3$ ) has been matched at the left to the SC curve, choosing the amplitude factor so that its integral over one or two grid intervals will be equal to the corresponding SC charge (i.e., to the corresponding length on the SC rectangle). Then, a sample has been chosen were a good match was observed between SC and the FD curve, and this was not difficult in a somewhat long interval. The final capacitance result has been computed summing up the formal integral of the Van Bladel exponent, from the origin to the above crossover sample, and an integral from this point to the end of the conductor side (trapezium rule). Similar results were obtained 
when the Van Bladel model was directly matched to the FD or to the SC curve at the above point of very similar ordinates.

The results for the capacitance value computed for the internal conductor are reported in Table 1. The numbers in the first row are the number of meshes (or grid intervals for SC); the second and third rows are respectively the percent errors observed in the uncorrected FD capacitances (already summarized in the previous section) and in the Van Bladel corrected ones.

Table 1. Percent errors for the uncorrected and corrected $C_{\text {int }}$

\begin{tabular}{c|c|c|c|c}
\hline \% error & 256 & 128 & 64 & 32 \\
\hline FD & -1.34 & -2.10 & -3.29 & -5.10 \\
\hline corr. FD & -0.05 & -0.28 & -1.03 & -3.58 \\
\hline
\end{tabular}

We see that the correction is very effective for large numbers of mesh points, and not so effective for smaller. However, the crossover point has been blindly chosen in proportion to the mesh numbers and no further effort was spent for these cases.

The a posteriori correction of $C_{\text {int }}$ has no effects on the other results of the FD calculation. A question is rising quite naturally, whether the gained information can suggest any way to build-in corrections in the SOR procedure itself.

A first idea is perhaps to supply the mesh with the lack of current above shown. A simple way can be to define at the boundary of the mesh Poisson's instead of Laplace's five point equations, and to add to the usual terms a fifth contribution obtained from the differences between the SC and the FD potential observed on each node of the grid adjacent to the internal conductor. As the potentials on the boundary are not involved in the SOR, we can expect the major effects in the direction normal to it.

Some experiments have been attempted, and some mitigation of errors has been found. Probably this is not exceeding some academic interest and, in any case, the interest is largely laying on the possibility of betting on reliable charge density distributions, to be blindly assumed. Sample results are reported in Table 2.

Table 2. Percent errors of various characteristics, before and after correction of the SOR process

\begin{tabular}{l|c|c|c|c}
\hline \# of intervals & 256 & 128 & 64 & 32 \\
\hline$\%$ err., $\boldsymbol{C}_{\text {ext }}$ FD & -0.020 & -0.052 & -0.132 & -0.335 \\
\hline \% err., $\boldsymbol{C}_{\text {ext }}$ FD corrected & -0.022 & -0.006 & -0.037 & -0.144 \\
\hline$\%$ err., $\boldsymbol{C}_{\text {int }}$ FD & -1.337 & -2.103 & -3.291 & -5.104 \\
\hline$\%$ err., $\boldsymbol{C}_{\text {int }}$ FD corrected & -0.137 & -0.714 & -1.641 & -3.271 \\
\hline$\%$ err., $\boldsymbol{C}_{\text {ext }} / \boldsymbol{C}_{\text {int }}$ & 1.357 & 2.154 & 3.418 & 5.421 \\
\hline$\%$ err., $\boldsymbol{C}_{\text {ext }} / \boldsymbol{C}_{\text {int }}$ corr. & 0.134 & 0.721 & 1.678 & 3.410 \\
\hline
\end{tabular}

In this table, the errors are reported with large numbers of figures only to better appreciate their trends, and rows have to be compared in pairs. The results have been obtained with a Poisson term equal to 0.7 times the difference in the above quoted potential. This was a good choice, but no deep investigation has been performed about the causes. As expected, the cor- 
rection has no influence on the capacitance computed on the exterior conductor $\left(C_{\mathrm{ext}}\right.$ in the Table), but it has significant effect on the capacitance computed on the interior one $\left(C_{\text {int }}\right)$. Errors are lowered for about 10 times for large numbers of mesh cells, but the effect is vanishing at small numbers. The ratio $C_{\text {ext }} / C_{\text {int }}$ follows this trend. We remark that the Van Bladel charge densities have only indirect influence on the table data: after introducing the additional term in the Poisson equations at the boundary, the conductor fluxes are computed exactly as in the SOR uncorrected procedure.

\section{A stripline example}

This section will be devoted to the analysis of the charge densities on a shielded dielectricsuspended stripline and to the conductor capacitance. The geometry of the structure is shown in Fig. 5, and it is useful both for the analysis of a coaxial line and for the odd mode of a pair of lines in quasi-TEM propagation mode.

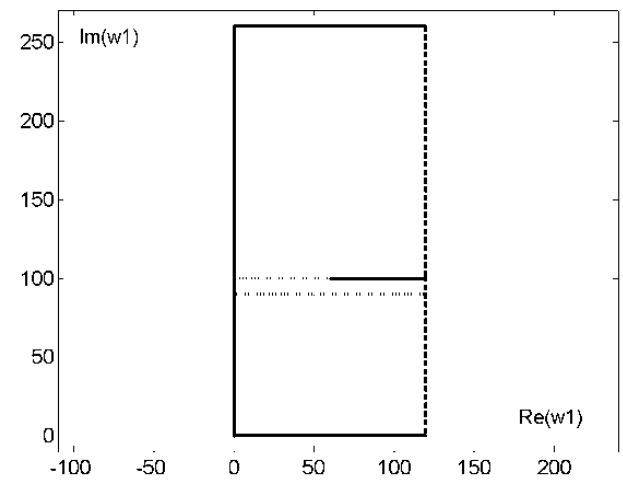

Fig. 5. The half section of a shielded dielectric suspended stripline. Coordinates in arbitrary units; dashed lines indicate Neumann walls; dotted lines the dielectric interfaces

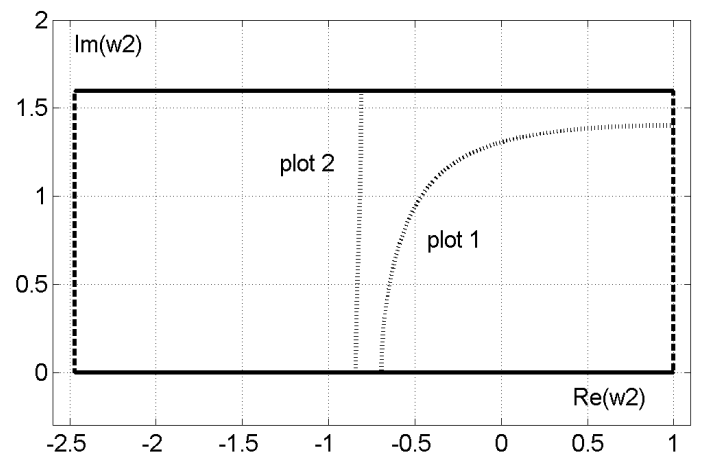

Fig. 6. The SC map into a rectangle of the stripline geometry of Fig. 5, with se same meaning of dashed and dotted lines. Coordinates in arbitrary units 
The SC map of the geometry is shown in Fig. 6, where the maps of the dielectric interfaces are indicated as plot 1 and plot 2.

As in the case of the L-shaped domain, the capacitance of the line can be immediately derived in homogeneous dielectric from the aspect ratio of the rectangle, and the equipotential lines in the original domain of Fig. 5 can be obtained by means of backward mapping horizontal straight lines in the rectangle of Fig. 6 . In the inhomogeneous dielectric case, accurate evaluation of the capacitance and of the charge densities on the conductors can be obtained by means of straightforward application of standard FD procedure to the rectangle, using suitable equations at the mapped dielectric interfaces.

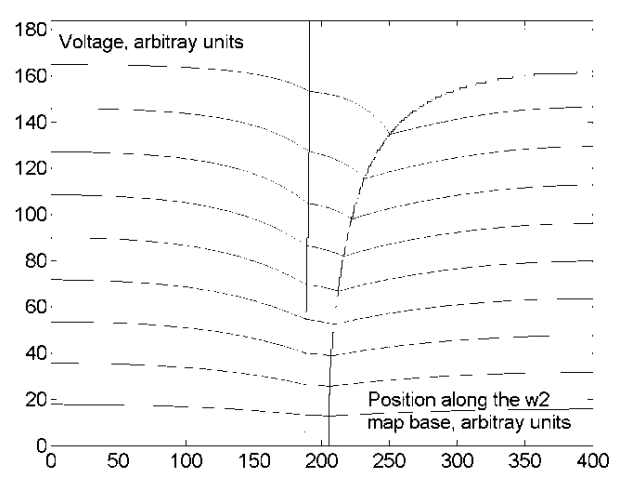

Fig. 7. Some equipotential lines computed in the rectangle of Fig. 6 , inhomogeneous dielectric $\left(\varepsilon_{r}=9.5\right)$, with the dielectric interfaces

Some equipotential lines computed in the rectangle of Fig. 6 are shown in Fig. 7.

To test the usefulness of the Van Bladel's model for the thin stripline of Fig. 5 in the homogeneous dielectric case, it will be sufficient to consider the charge density on the upper side of the strip, which is shown in Fig. 8: in the lower side a similar behaviour will be found.

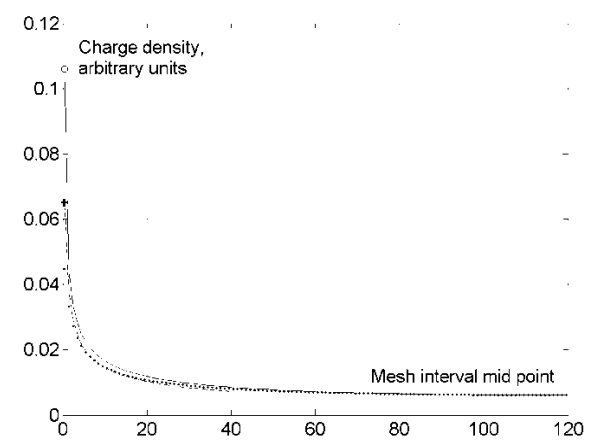

Fig. 8. Charge densities computed for the upper side of the stripline in Fig. 5 in homogeneous dielectric: dots: FD in the original geometry; solid line and circle: SC; dashed line and cross: Van Bladel model

The SC capacitance in air is $C_{0 \mathrm{SC}}=1.922490 \cdot 10^{-11} \mathrm{~F}$. The value obtained from FD in the original geometry with a mesh of dimensions $240 \times 520$ was $C_{\text {ground }}=1.926564 \cdot 10^{-11} \mathrm{~F}$ when 
computed on the shield, with very good agreement to SC because of the shape of the geometry in Fig. 1 and of the selected dimensions. Instead, computing the capacitance on the strip led to an error of about $-6 \%$.

However, Fig. 8 shows similar behaviours for the SC and for the FD curves, and this encouraged to blindly match the Van Bladel model to the FD curve at the $40^{\text {th }}$ interval along the strip. This way the integral of the charge was increased by some 7\%. Matchig the model at the $30^{\text {th }}$ or at the $50^{\text {th }}$ sample the results were not very different: about $6 \%$ and about $8 \%$. However, the agreement observed at the left of the figure was somewhat worse than in the case of the L-shaped domain, as seen in the figure.

Cosidering that in any case the agreement with the right hand of the FD curve would remain good, this suggested to look again to the behaviour of the SC curve, matching the Van Bladel model to it at the $20^{\text {th }}$ sample, but again the results were not satisfactory.

Perhaps the Van Bladel exponent itself has to be better matched to the true charge density provided by conformal mapping; in any case, it seems that blind exploitation of the model would often mitigate the FD errors but not always achieve very accurate results.

The capacitance computed via SC+FD in the rectangle in Fig. 6 was $C_{\mathrm{SCFD}}=3.289427 \cdot 10^{-11} \mathrm{~F}$, and $3.285440 \cdot 10^{-11} \mathrm{~F}$ after taking into account the small difference between the true aspect ratio of the rectangle in Fig. 6 and the aspect ratio of the discrete FD mesh. Because of the large number of mesh cells $(400 \times 184)$, the difference between these values is small, about only $0.1 \%$, and they are in agreement with FEM results obtained with large number of elements, with differences limited to about $1 \%$.

The charge densities computed on the mesh for the stripline and for the shield electrode are shown in Fig. 9, and their behaviour are well explained by the shapes of the dielectric interfaces mapped in the rectangle: see plot 1 and plot 2 in Fig. 6 . We remark the discontinuity at the dielectric interface when traveling along the strip from the upper side (facing air) to the bottom (facing the dielectric).

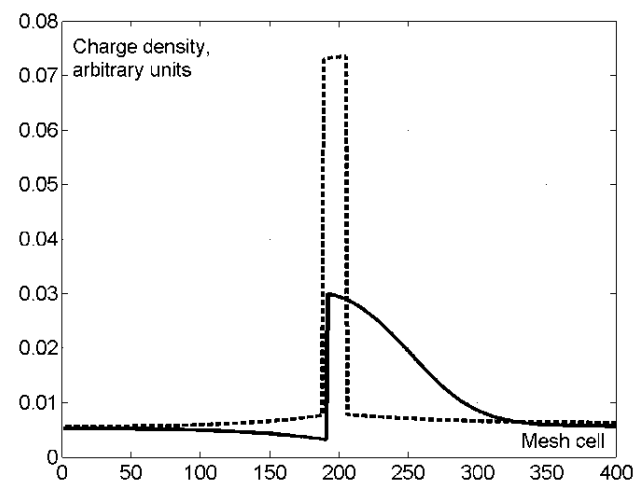

Fig. 9. Charge density on the FD mesh built on the rectangle of Fig. 6. Solid line: upper conductor side (the half stripline section in Fig. 5); dashed line: bottom conductor side (the shield) in Fig. 5

Before attempting to exploit a Van Bladel model based as before on charge densities on the original geometry, it is necessary to map back the abscissas of Fig. 9. Of course, this was 
easily done performing a side scanning similar to that used to compute the charge densities in the homogeneous case: the result for the right half part of the solid line of Fig. 9 is shown in Fig. 10. Here, the SC+FD charge density on the stripline is compared to that obtained from FDs working directly in the original geometry.

We observe in the figure not only the usual failure of the FD computation to represent the behaviour of the charge density in close proximity to field singularities, but also a notable difference along more than one third of the strip width. At first sight, to match here a simple exponential model will be very difficult.

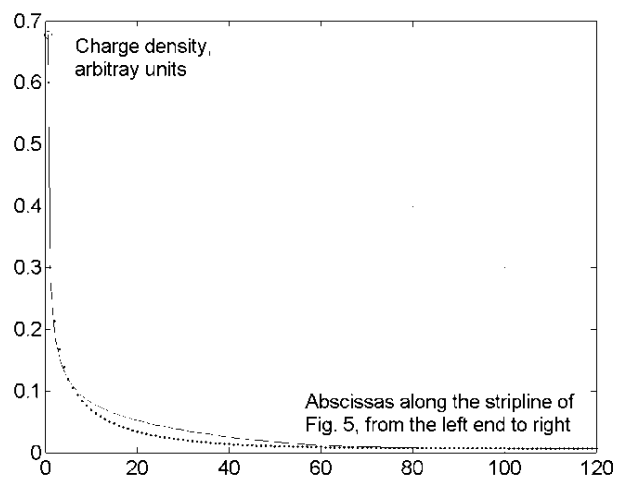

Fig. 10. The SC+FD charge density remapped to the plane of Fig. 5 (solid line) compared to that obtained from FD directly in this plane (dotted line)

\section{Conclusions}

A quantitative analysis has been performed on the accuracies obtained in simple cases from FD procedures, by means of Schwarz-Christoffel maps followed in inhomogeneous dielectric by FD calculations on rectangular meshes. An attempt has been made to evaluate the possibility of correcting faulty behaviours of charge densities by means of Van Bladel models, more or less blindly or with the help of true behaviours obtained from conformal maps.

\section{References}

[1] Driscoll T.A., Trefethen L.N., Schwarz-Christoffel Mapping, Cambridge University Press, 9-30 and 70-74 (2002).

[2] Costamagna E., Di Barba P., Mognaschi M.E., Savini A., Fast algorithms for the design of complexshape devices in electromechanics, Computational Methods for the Innovative Design of Electrical Devices, Springer, pp. 61-65 (2010).

[3] Costamagna E., A new approach to standard Schwarz-Christoffel formula calculations, Microwave and Optical Technology Letters 32(3): 196-199 (2002).

[4] Costamagna E., Di Barba P., Savini A., A kinematic approach to the optimal shape synthesis of electric fields, Electrical Review, ISSN 0033-2097, 88(7b): 90-93 (2012).

[5] Alfonzetti S., Costamagna E., Fanni A., Computing capacitances of vias in multilayered boards, IEEE Transactions on Magnetics 37(5): 3186-3189 (2001). 
[6] Costamagna E., A simple procedure to optimize small radius rounded corners obtained from Schwarz-Christoffel conformal transformations, IEEE Transactions on Magnetics 51(3): 3186-3189 (2015).

[7] Costamagna E., Di Barba P., Palka R., Field models of high-temperature superconductor devices for magnetic levitation, Engineering Computations 29(6): 605-616 (2012).

[8] Van Bladel J., Singular Electromagnetic Fields and Sources, Clarendon Press, Oxford, Chapt. 4 (1991).

[9] Van Bladel J., How electrically sharp is a needle?, IEEE Antennas and Propagation Magazine, 45(5): 118-122 (2003).

[10] Costamagna E., Conformal mapping and field singularities in perfectly conducting wedge and rotational symmetry structures, Microwave and Optical Technology Letters 24(3):191-195 (2000). 